ACCEPTED MANUSCRIPT

\title{
Fully printed all-polymer tattoo/textile electronics for electromyography
}

To cite this article before publication: Eloise Bihar et al 2018 Flex. Print. Electron. in press https://doi.org/10.1088/2058-8585/aadb56

\section{Manuscript version: Accepted Manuscript}

Accepted Manuscript is "the version of the article accepted for publication including all changes made as a result of the peer review process, and which may also include the addition to the article by IOP Publishing of a header, an article ID, a cover sheet and/or an 'Accepted Manuscript' watermark, but excluding any other editing, typesetting or other changes made by IOP Publishing and/or its licensors"

This Accepted Manuscript is @ 2018 IOP Publishing Ltd.

During the embargo period (the 12 month period from the publication of the Version of Record of this article), the Accepted Manuscript is fully protected by copyright and cannot be reused or reposted elsewhere.

As the Version of Record of this article is going to be / has been published on a subscription basis, this Accepted Manuscript is available for reuse under a CC BY-NC-ND 3.0 licence after the 12 month embargo period.

After the embargo period, everyone is permitted to use copy and redistribute this article for non-commercial purposes only, provided that they adhere to all the terms of the licence https://creativecommons.org/licences/by-nc-nd/3.0

Although reasonable endeavours have been taken to obtain all necessary permissions from third parties to include their copyrighted content within this article, their full citation and copyright line may not be present in this Accepted Manuscript version. Before using any content from this article, please refer to the Version of Record on IOPscience once published for full citation and copyright details, as permissions will likely be required. All third party content is fully copyright protected, unless specifically stated otherwise in the figure caption in the Version of Record.

View the article online for updates and enhancements. 


\title{
Fully printed all-polymer tattoo/textile electronics for electromyography
}

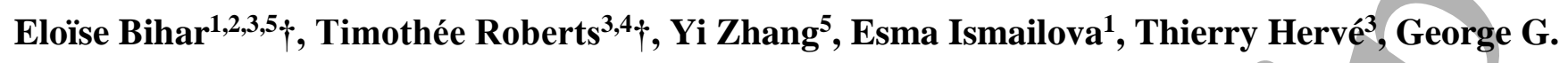
${\text { Malliaras }{ }^{1} \uparrow \dagger \text {, Jozina B. De Graaf }}^{4}$, Sahika Inal ${ }^{5 *}$ and Mohamed Saadaoui ${ }^{2}$

${ }^{1}$ Department of Bioelectronics, Ecole Nationale Supérieure des Mines, CMP-EMSE, MOC, 13541

Gardanne, France

${ }^{2}$ Department of Flexible Electronics, Ecole Nationale Supérieure des Mines, CMP-EMSE, MOC, 13541 Gardanne, France

${ }^{3}$ Microvitae Technologies, Hôtel Technologique, Europarc Sainte Victoire, Bâtiment 6, Route de Valbrillant, Meyreuil, 13590, France

${ }^{4}$ Institut des Sciences du Mouvement, CNRS, Aix Marseille Université, ISM UMR 7287, 163, Avenue de Luminy, CP 910, 13288 Marseille Cedex 9, France

${ }^{5}$ Biological and Environmental Sciences and Engineering Division, King Abdullah University of Science and Technology (KAUST), Thuwal 23955, Kingdom of Saudi Arabia

*Corresponding author: sahika.inal@kaust.edu.sa

$\dagger$ These authors contributed equally to this work.

$\dagger \dagger$ Current address: Electrical Engineering Division, University of Cambridge, Cambridge CB3 0FA, United Kingdom

\section{Keywords}

Inkjet printing, organic electronics, tattoo, textile, electrophysiology

\begin{abstract}
Driven by the ever-growing needs for developing low cost, easy-to-use, noninvasive diagnostic tools, biomedical devices that can be integrated on human skin or textiles have begun to emerge. These "wearable" devices should couple electronics directly to the human skin and detect a variety of biologically relevant signals such as the neuromuscular activity. In this work, we develop a simple, low cost and customizable device to perform electromyography (EMG) measurements based on electronics
\end{abstract}


fabricated on a tattoo paper. The electrodes are based on the conducting polymer poly $(3,4-$ ethylenedioxythiophene) doped with polystyrene sulfonate (PEDOT:PSS) and inkjet-printed on the conformable tattoo paper. Addressing the integration challenge common for such flexible electronic devices, we connect the tattoo electrodes to the acquisition system through a textile in the form of a wristband comprising of printed PEDOT:PSS contacts. While the textile wristband conforms around the "tattooed" skin, it enables a reliable contact with the electrodes beneath. We show that this tattoo/textile electronics platform is able to monitor the biceps activity of the arm during muscle contractions lasting for a period of seven hours, with comparable performance to conventional biopotential electrodes and without the use of gels or expensive metallic materials. Combining the tattoo electronics with the electronic textile improves the communication of skin-like electrodes with external electronics, renders a reliable and versatile system for detecting biopotential signals critical for myoelectric prosthesis, muscle injury prevention and/or detection.

\section{Introduction}

One of the greatest endeavors of current medical diagnostics research is to develop devices that can record vital signals from the body in real time whilst being minimally invasive [1]. The sensor technologies thus move from lab-on-chip platforms to wearable electronics, indicating that sensors should be integrated with the skin or clothes that surround the skin, allowing for personal health monitoring as well as drug delivery systems [2-8]. Electrically interfacing the skin with electrodes for monitoring the electrical activity related to functioning of cells is a well-established practice in clinic, albeit performed with bulky rigid electronics that are not comfortable to wear. For instance, standard devices to record muscle activity, i.e. electromyography (EMG), are nonpolarizable $\mathrm{Ag} / \mathrm{AgCl}$ electrodes which are applied on the skin typically with gels that allow for ion/water penetration and therefore reduce skin/electrode impedance. The performance of these electrodes depends on the gel-material and as such they pose several limitations including discomfort, allergies or irritation when in contact with the human skin, as well as limited stability 
related to gel drying out during long-term applications [9]. New materials and concepts have emerged in order to address these aforementioned limitations, aiming to develop devices that can adhere seamlessly to skin as well as adapt to its movements. Conformable electrodes based on smart textiles are prime devices generated to fulfill these requirements [10-13]. Someya and coworkers, for instance, demonstrated new conductors made of highly stretchable materials composed of Ag flakes mixed with fluorines. These materials could be integrated into garments via screen printing and used for recording electrophysiological signals [12]. Graphene has been another material integrated with nylon fabrics [13]. In parallel to the development of smart textiles, a new generation of deyices that can be fully conformable, known as electronic skin or e-skin, $[7,14,15]$ has emerged. The pioneering work in this field incorporated electrophysiology, temperature and strain sensors made of gold, thin silicon, gallium arsenide in the form of nanoribbons and nanomembranes [15]. These materials were deposited directly on the skin by dissolving a water soluble film of polyvinyl alcohol, leaving the electronics adhered to the skin by van der Walls interactions [15]. Recently Bao's team proposed a new route for the fabrication of "epidermal" circuitry comprising transistors arrays, based on stretchable polymer materials such as carbon nanotubes and conjugated polymer/elastomer mixtures, and developed integrated touch-sensors able to detect the footprint of an artificial ladybug [8]. These devices were transferred to the skin using a water-soluble sacrificial layer as well. A promising substrate material for device integration has in fact been the commercially available tattoo paper. If the electronics can be integrated on the paper, it can be transferred to the skin by dissolving the water-soluble sacrificial layer (typically made of starch-dextrin) of the paper. As such, the electronics conforms onto the complex features of the skin and represents an ideal disposable system for performing noninvasive daily diagnostics of a variety of biomarkers. These tattoo integrated electronic platforms exhibit excellent skin conformation, resistance to deformation and good biocompatibility with the human skin $[2,16-18]$.

Organic electronic materials have come to the fore as promising materials in interfacing the tissue due to the mechanical softness and biocompatibility as well as the low impedance of their films [19]. Amongst 
these materials, poly(3,4-ethylenedioxythiophene) doped with polystyrene sulfonate (PEDOT:PSS) has been the standard organic electronic coating, owing its popularity mostly to its electrochemical stability (no degradation in aqueous environments rich in oxidative species) and being readily available as a dispersion in water through commercial vendors [20]. Another reason for the current popularity of PEDOT:PSS is that its dispersion can be processed through various means, allowing for integration into a wide range of existing fabrication procedures. Co-solvents, secondary dopants, and crosslinkers are often added to the dispersion to modulate the film conductivity, mechanical properties as well as viscosity so that it is ideal for printing and coating over large areas [21-27]. All these properties render PEDOT:PSS an ideal material to cast on unconventional substrates such as the tattoo paper. Zucca et al reported different techniques for the fabrication PEDOT:PSS based tattoo nanosheet electrodes. These involve spin casting the polymer on a commercial tattoo paper and inkjet printing a $\mathrm{NaClO}$ solution for the subtractive patterning of the tattoo [28], as well as coating poly(D,L-lactic acid) and PEDOT:PSS on a polypropylene substrate with a gravure roll [29]. In both cases, after transferring the tattoo electrode on the skin, EMG signals could be successfully recorded. The integration of interconnects is however challenging for tattoo electronics and needs to be considered for stability and use for long term measurements. Bareket et al developed tattoo electronics based on screen printed carbon electrodes and plasma polymerized PEDOT:PSS [30]. The device recorded EMG signal for 3 hours, however the overall thickness of the

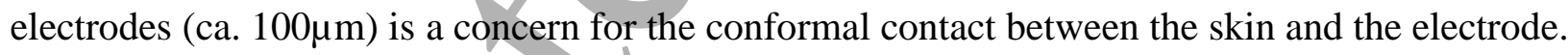

For these disposable and organic electronics-based tattoo electrodes to be ever commercialized, the fabrication should be cost and time effective. Solution processing technologies such as spraying techniques [31], screen printing [32,33], or inkjet printing [26,34] not only involve a small number of fabrication steps, but are also compatible with large-area flexible substrates. Inkjet printing, in particular, allows for the digital fabrication of customizable designs at atmospheric conditions, with patterns that can be easily modified on demand, ideal for fabricating diagnostic devices on flexible substrates [35,36]. A recent work of Ferrari et al demonstrated PEDOT:PSS electrodes printed on a tattoo paper which could 
measure muscular activity with high precision on limbs and face [37]. The electrode allows for hair growth underneath, a great advantage over conventional systems promising for long-term recordings on areas with high hair density. For practical applications, however, where the measurements are intended to be carried on the skin for longer periods of time, the integration of the tattoo with the acquisition system remains as a challenge that needs to be addressed. Design solutions should exclude adhesives, insulating layers as well as metallic interconnects in order to reduce fabrication costs and involve a user-friendly interface for these disposable systems. It is also important that all the fabrication procedure is accomplished in one printing step.

In this work, we present a simple process for the fabrication and integration of a fully inkjet-printed e-skin device based on tattoo technology. Using PEDOT:PSS both/as the active electrode as well as contact material, we record high quality EMG signals from the arm's biceps over 7 hours. Replacing conventional electrical wires or any metal contact fabricated via evaporation which limit the feasibility of e-skin technologies, we utilize PEDOT:PSS contacts printed on a conformable textile wristband. We characterize this "tattoo/textile electronics" system and investigate the performance of the devices compared to commercial electrodes used for EMG recordings. The thickness and roughness of the tattoo electrodes and electrical properties of the tattoo/textile electronics are evaluated. The tattoo/textile electronics-skin contact impedance at the relevant frequency range are measured. With the textile wristband conforming on the skin and the intimate adhesion of the tattoo, we are able to record stable EMG signals with comparable performance to conventional wet (gelled) $\mathrm{Ag} / \mathrm{AgCl}$ electrodes. The proposed platform paves the way towards easy-to-fabricate and use, conformable, all-polymer biopotential electrodes that can be utilized in a wide range of diagnostics and as well as therapy applications.

\section{Experimental}

Fabrication of PEDOT:PSS based electronics on tattoo and textile: PEDOT:PSS ink was prepared using a mixture containing PEDOT:PSS dispersion (conducting polymer, Heraeus, Clevios PH1000), $20 \mathrm{wt} \%$ ethylene glycol (EG, co-solvent, Sigma Aldrich) and $0.8 \mathrm{wt} \%$ glycidoxypropyltrimethoxysilane (GOPS, 
crosslinker, Sigma Aldrich). While EG increases the conductivity of the films deposited, GOPS prevents their delamination during the transfer of the tattoo onto the skin. In this mixture, we also included $0.3 \mathrm{wt} \%$ of commercially available surfactants and a combination of organic solvents to render rheological properties of the ink suitable for inkjet process without compromising softness and electrical conductivity. A Dimatix DMP-2800 inkjet printer was used to fabricate the tattoo/textile electronics. $1 \mathrm{~cm}^{2}$ square pattern of PEDOT:PSS (single layer) was printed on a commercially available temporary tattoo sheet (Tattoo 2.1, the Magic Touch Ltd., 123 Applications). The sheet is composed of a single decal transfer paper (including 3 layers : a paper carrier, a starch-dextrin water soluble sacrificial layer, and a layer of ethylcellulose (ca. 400nm in thickness). For the wristband, we printed up to 8 layers of PEDOT:PSS ink on a commercially available satin ribbon (Ideatiss). The metallic snaps buttons were installed on the ribbon via a snap plier. The drop spacing for all printing procedures was $15 \mu \mathrm{m}$. Once the ink was printed, the samples were placed in a conventional oven to cure the films at $110^{\circ} \mathrm{C}$ for $60 \mathrm{~min}$. The tattoo electrodes were transferred to the skin (no pre-treatment) by dissolying the water-soluble sacrificial layer and removed by washing the exposed skin with soap and water.

Electrical characterization of the tattoo electrodes: A four-point probe system (Jandel) was used to determine the sheet resistance of PEDOT:PSS films. We used a mechanical profilometer (Ambios Technology) to estimate the thickness of a single PEDOT:PSS layer printed on the substrates. The electrical resistance of the PEDOT:PSS contact on the textile as well as the one between the tattoo electrode and the textile contact was measured using a digital Multimeter (Amprobe 37XR-A Tru-rms).

Electrode-skin contact impedance measurements were performed as shown in Figure 2. The working electrode (e.g. the tattoo/textile electronics) and the counter electrode (wet $\mathrm{Ag} / \mathrm{AgCl}, \mathrm{Ambu}$ blue sensor) were placed on the forearm of a volunteer, keeping $3 \mathrm{~cm}$ distance from each other. The reference electrode (wet $\mathrm{Ag} / \mathrm{AgCl}, \mathrm{Ambu}$ blue sensor) was placed on the elbow. A sinusoidal potential of $10 \mathrm{mV}$ was applied at the working electrode using a potentiostat (Metrohm Autolab B.V.) with no constant differential voltage on the skin. The impedance was screened for a frequency range between 0.1 and $1000 \mathrm{~Hz}$ and the data 
were analyzed using NOVA software. We evaluated the electrical stability of the tattoo electrode upon mechanical deformation. We transferred the tattoo across the distal phalange of the index finger that is exposed to multiple flexions/extensions. Two copper tapes adhering to the printed PEDOT:PSS electrode on opposite edges were used to establish electrical connection with the acquisition system. The flexion/extension was repeated 250 times at $30^{\circ}$ and at $90^{\circ}$ angle of closure. The resistance of the electrode was measured at every 10 repetitions.

EMG recordings and data acquisition: Three able-bodied volunteers ( 2 males, 1 female, ages between 25 and 40 years old) participated in this study with informed consent acquired before conducting the experiments. All participants took part for the long-term EMG recordings and one volunteered for the measurement of the electrode-skin impedance. All protocols and procedures were approved by the direction of research of the Ecole des Mines de St. Etienne. Two tattoo electrodes were placed $2 \mathrm{~cm}$ apart from each other at the center of the biceps of the left arm. The contacts printed on the textile wristband were placed on top of the tattooed areas of the skin in order to connect the recording and counter electrodes to the acquisition system. 2 wet $\mathrm{Ag} / \mathrm{AgCl}$ electrodes (Metrohm Autolab B.V.) were placed on the biceps of the right arm. In order to have comparable signals, the volunteer was asked to lift $1 \mathrm{~kg}$ of a standard weight with each arm simultaneously. A ground electrode was placed on the right leg. For each recording, the experiment has been repeated at least 6 times, and the data were collected once in every 3 hours over 7 hours $\left(T_{0}=1 h, T_{0}+3 h\right.$ and $\left.T_{0}+6 h\right)$. EMG data were acquired using A RHD2216 chip from Intan Technologies. The data were recorded with 16 -bit resolution and a sampling rate of $1.1 \mathrm{kHz}$ per channel and then transferred to a PC through an USB cable. More information can be found in the datasheet of the RHD2216 chip from Intan Technologies. The on-chip filters were set as follows: the first order high pass was set at $10 \mathrm{~Hz}$ and the third order low pass filter was set at $300 \mathrm{~Hz}$.

Processing of EMG data: We used a customized Labview software to filter the signal and calculate the signal-to-noise-ratio (SNR). All recordings were treated with a first order Butterworth band-pass (10-300 $\mathrm{Hz}$. The SNR value of each recording was then calculated based on the following equation: 
$S N R(S r)=20 * \log \left(\sqrt{\frac{\sum_{i} S c_{i}^{2}}{\sum_{i} S r_{i}^{2}}}\right)$, where $\mathrm{S}_{\mathrm{c}}$ is a portion of $5 \mathrm{~s}$ of the signal during a contraction and $\mathrm{S}_{\mathrm{r}}$ is a portion of $5 \mathrm{~s}$ of the signal during a resting position.

\section{Results and Discussion}

Figure 1a shows a layer schematic of the tattoo electronics integrated with the textile contacts. The tattoo includes one layer of PEDOT:PSS ink inkjet-printed onto the temporary tattoo paper. PEDOT:PSS coating increases the roughness (RMS) of the substrate from $1.35 \mathrm{~nm}$ to $3.77 \mathrm{~nm}$ (Figure S1). The electrical conductivity of a $95 \pm 5 \mathrm{~nm}$ thick printed PEDOT:PSS electrode (single layer) is $240 \pm 10 \mathrm{~S} / \mathrm{cm}$. The thickness and the associated sheet resistance can be typically controlled by varying the number of printed layers of PEDOT:PSS. However, we find the tattoo substrate not compatible with printing multiple layers of aqueous ink due to instability of the sacrificial layer in aqueous. We estimated the conductivity of a single layer of the film to be adequate to acquire EMG signals. Following the fabrication, the electronics printed on the paper was transferred onto the skin by simply wetting the tattoo transfer paper and gently pressing the tattoo against the skin. As the transfer paper is wetted, it dissolves and the conducting polymer pattern remains on the skin. As inkjet offers freedom of form and design, different electrode patterns can be achieved (Figure 1b). The electrode conforms well to the skin and is in fact reported to enter deeply inside the creases of the skin [37] (Figure 1c). As the skin is compressed or stretched due to muscle movements, the electrodes move with the skin, acting as its natural component (Figure 1c). The electrodes tattooed on the skin have an intimate contact with the tissue and by using the conducting polymer which has a Young's modulus of ca. 150MPa in wet state [25], similar to that of skin ca. 2kPa-500 MPa [38], we overcome the inherent mechanical mismatch (Figure 1d). 


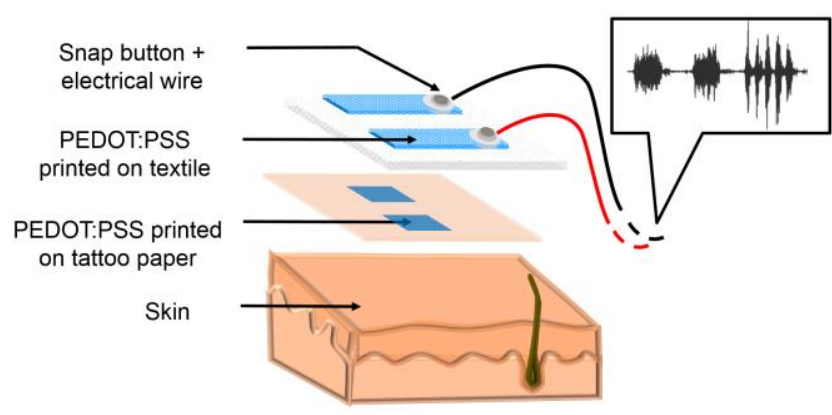

(a)
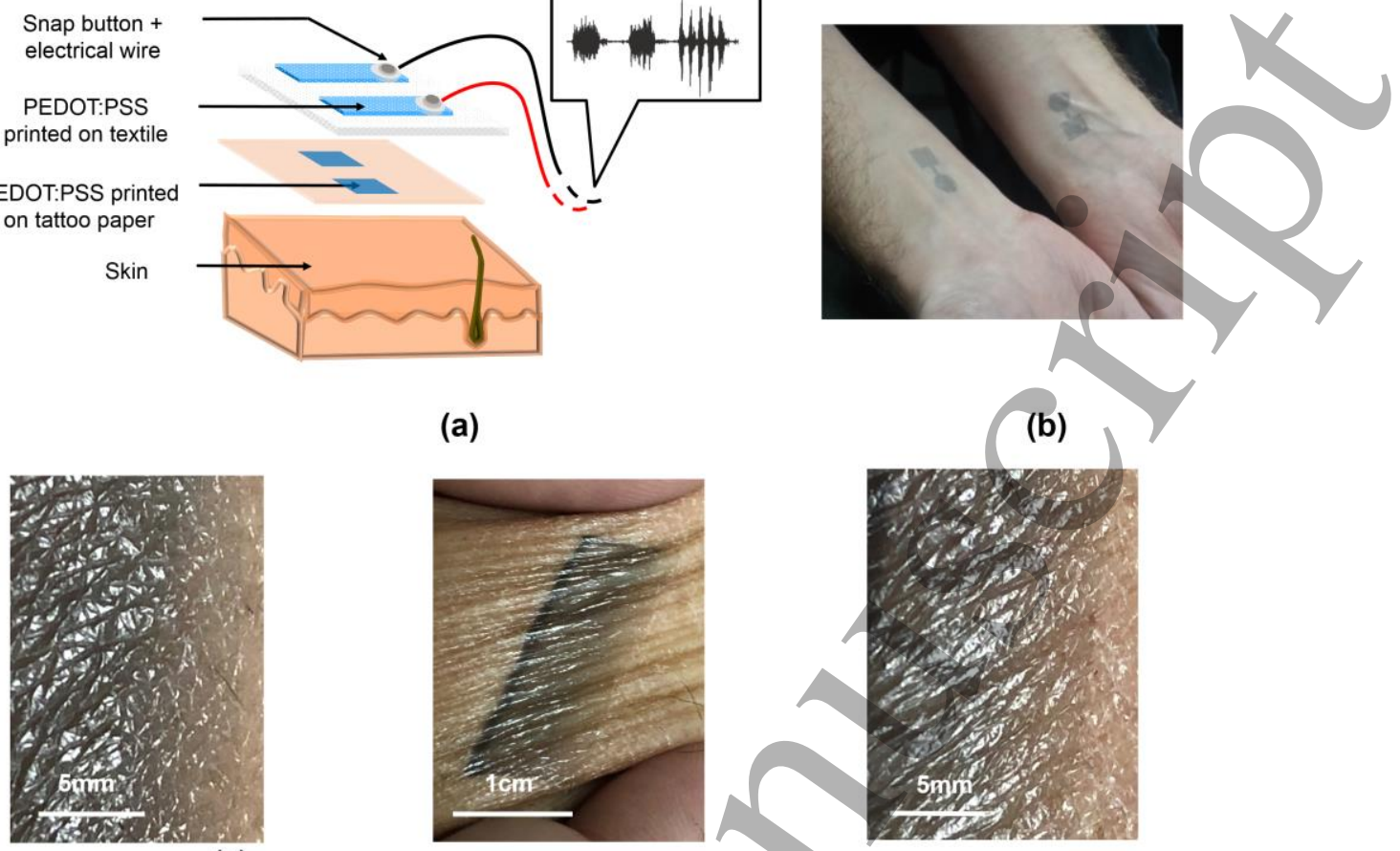

(b)

(c)

(c) (d) (e)

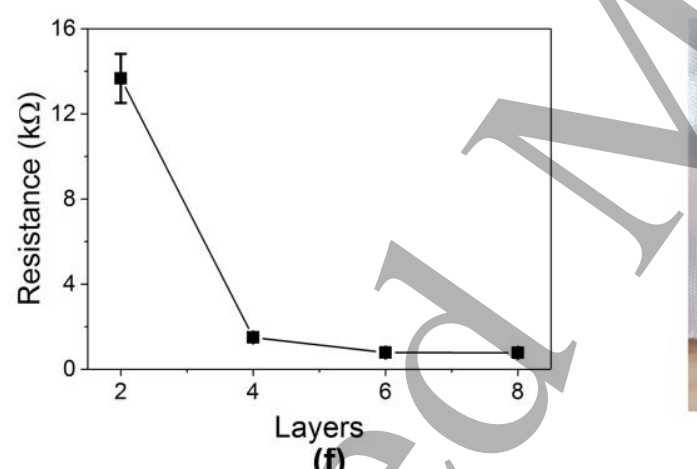

(f)

(g)

Figure 1. The architecture of tattoo-textile electronics (a) Schematic of the tattoo/textile electronics. The PEDOT:PSS electrode printed on the tattoo paper is transferred to the skin. The textile containing the printed conducting polymer contacts is wrapped around the tattooed electrode on the skin, connecting the tattoo to the acquisition system. (b) Photograph of customized printed tattoo electrodes deposited on the skin. (c, d, and e) Photographs of printed tattoo electrodes on the skin: undeformed (c), compressed several times (d), and after compression (e). The tattoo electrode shows a good resistance to mechanical deformation, with no cracks observed at the surface of the electrode. (f) Photograph of the PEDOT:PSS printed on the textile (6 layers) with the snap buttons integrated. (g) Electrical resistance of the printed PEDOT:PSS patterns on the textile $\left(1.5 \times 1.5 \mathrm{~cm}^{2}\right)$.

The conducting polymer was also printed on the textile wristband made of knitted soft fabric (i.e. satin) using the same printing protocol as for the tattoo paper (Figure 1f). In order to optimize the electrical performance of the contacts on the textile, we varied the number of the printed PEDOT:PSS layers 
$\left(1.5 \times 1.5 \mathrm{~cm}^{2}\right)$. The electrical resistance of the contacts decreased with the number of printed layers and reached a plateau with 6 layers of PEDOT:PSS (Figure 1g). The saturation is due to complete impregnation of the fabric with the polymer. Following the fabrication of electrodes, we inserted metal snap buttons to the textile on top of the conducting pattern. With the buttons attached, the textile was in the form of a wristband which could comfortably be wrapped around the target skin area where the tattoo electrodes were transferred and fixed using the snap button closure (Figure 1f). As such, we directly interface the tattooed electrodes with the contacts on the textile. The metal snaps enable to connect the tattoo electrodes to the acquisition system in order to record the electrophysiological activity. The electrical resistance between the tattooed electrodes on the skin and the metal snap on the textile is measured to be ca. $52 \pm 5 \mathrm{k} \Omega$ (Figure S2). This configuration obviates the need to use external wires in contrast to other studies with EMG electrodes relying on external connectors such as silver cotton wires [28], gold coated plastic foils [29], or polyimide films [30]. These contacts tend to cause unstable measurements due to motion artefacts and discomfort to the user, as well as their integration requires additional fabrication steps. In the tattoo/textile electronics, there is only one main electronic material, PEDOT:PSS, and it is deposited in a single step using one fabrication protocol.

We compared the electrode-skin contact impedance of the tattoo/textile electronics with the conventional wet (gelled) $\mathrm{Ag} / \mathrm{AgCl}$ electrodes, one hour after they have been in contact with skin (Figure 2a). Figure $2 \mathrm{~b}$ shows the configuration of the measurements on the forearm of a volunteer. The commercial electrodes exhibited impedance values two orders of magnitude lower than the tattoo/textile electronics at low frequency range (from $0.1 \mathrm{~Hz}$ to $100 \mathrm{~Hz}$ ). For frequencies above $100 \mathrm{~Hz}$, the impedance of the tattoo/textile electronics was one order of magnitude higher. The lower impedance of the commercial electrodes is due to the presence of the gel electrolyte applied between the $\mathrm{Ag} / \mathrm{AgCl}$ and the skin [39]. The tattoo/textile system, on the other hand, shows reduced impedance values for all the frequencies explored, as well as a smaller sample-to-sample deviation compared to the printed textile electrode only. At the frequency range where the significant EMG signals occur $(5-450 \mathrm{~Hz})$ [40], the tattoo/textile electronics 
display an impedance of ca. $1.83 \pm 0.01 \mathrm{M} \Omega$ at $5 \mathrm{~Hz}$ and $225 \pm 10 \mathrm{k} \Omega$ at $450 \mathrm{~Hz}$. Albeit with impedance higher than conventional electrodes, we and others postulate that the dry electrodes have the advantage of being more stable [41].

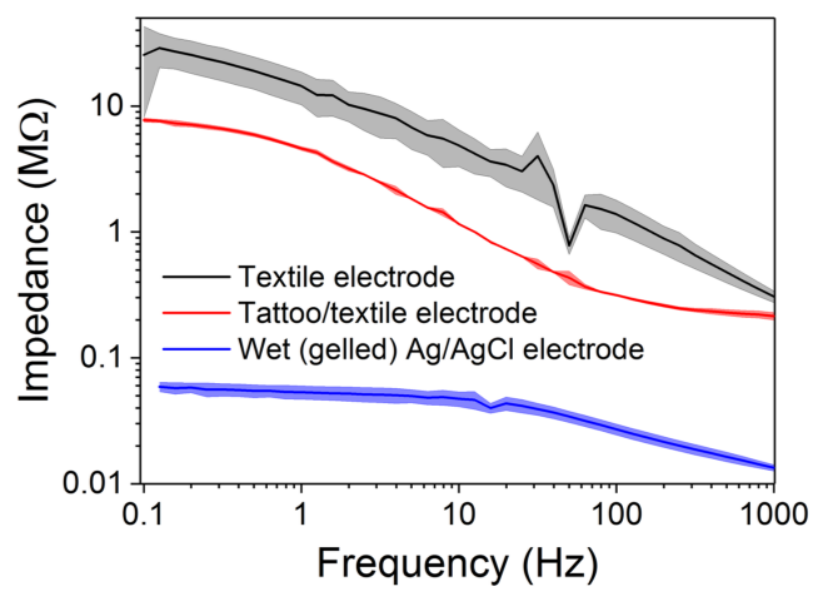

(a)

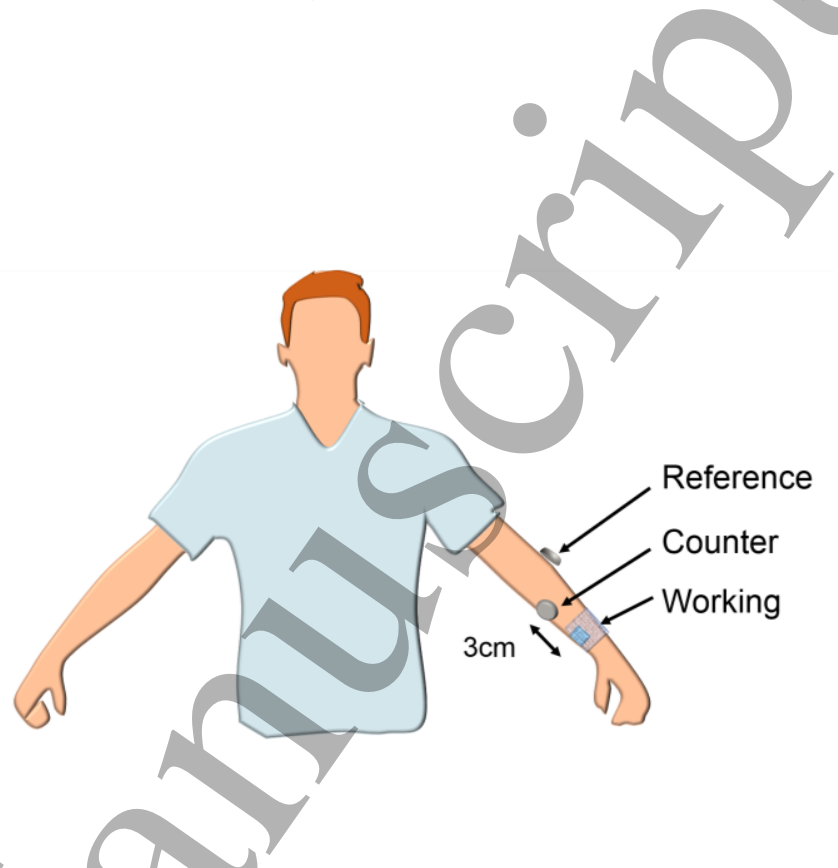

(b)

Figure 2. Electrical characterization of the electrodes (a) Electrochemical impedance spectra of the textile, tattoo/textile electronics and wet $\mathrm{Ag} / \mathrm{AgCl}$ electrodes after one hour of contact on the skin. The evaluation includes three devices and the shaded areas represent the standard deviation. (b) Schematic representation of the experimental set-up showing electrode positions on the forearm: working electrode is the textile, tattoo/textile electronics or wet $\mathrm{Ag} / \mathrm{AgCl}$ lectrodes, while counter and reference electrodes are wet $\mathrm{Ag} / \mathrm{AgCl}$.

In contrast to the rigid conventional electrodes, tattoo electrodes conform with the skin. In order to demonstrate the stability of the tattoo electrode upon bending, we measured its electrical resistance under deformation. The tattoo was deposited between the distal and intermediate phalanges of the index finger of the volunteer who was asked to perform repetitive flexions/extensions (Figure 3a and b). We recorded the electrical resistance of the electróde as the finger was subject to different bending angles $\left(30^{\circ}\right.$ and $\left.90^{\circ}\right)$ up to 250 cycles. While we do not observe a significant variation in the electrical resistance at a flexion to $30^{\circ}$, resistance increases slightly for flexion to $90 .^{\circ}$ This is attributed to the failure of the thick metallic adhesives used to contact the tattoo, proposed also by Zucca and colleagues [29]. In fact, we observe such cracks observed at the adhesive-tattoo interface after the experiments (Figure S3). In parallel, we examined the electrical stability of the contact printed on the textile electrode worn for a period of 7 hours on the 
arm wrist. We observed no changes in the polymer conductivity for this period, evidencing the stability of the textile contact.

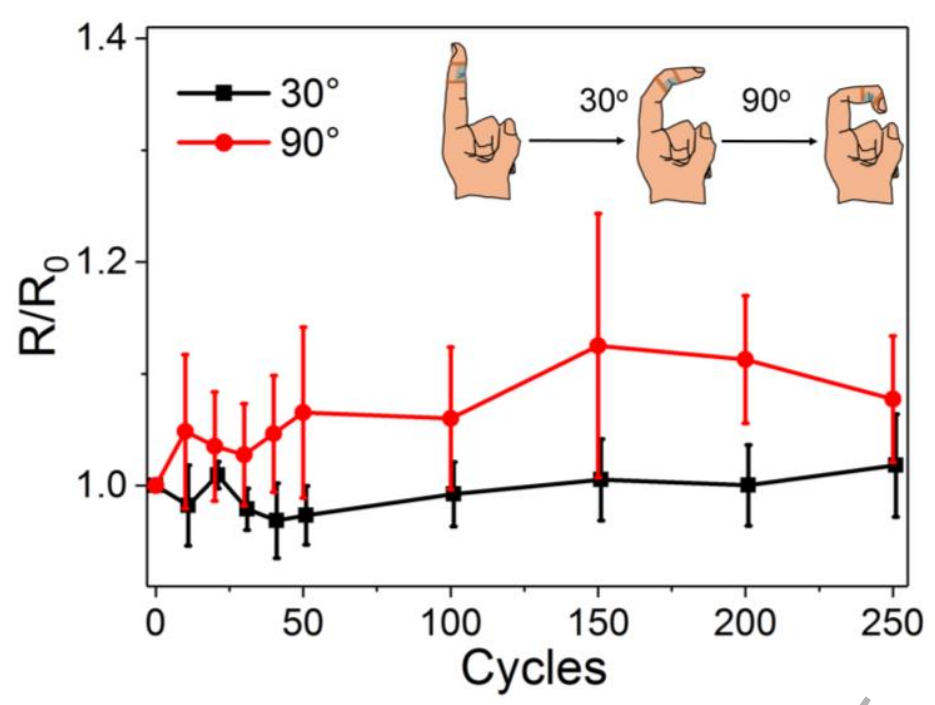

(a)

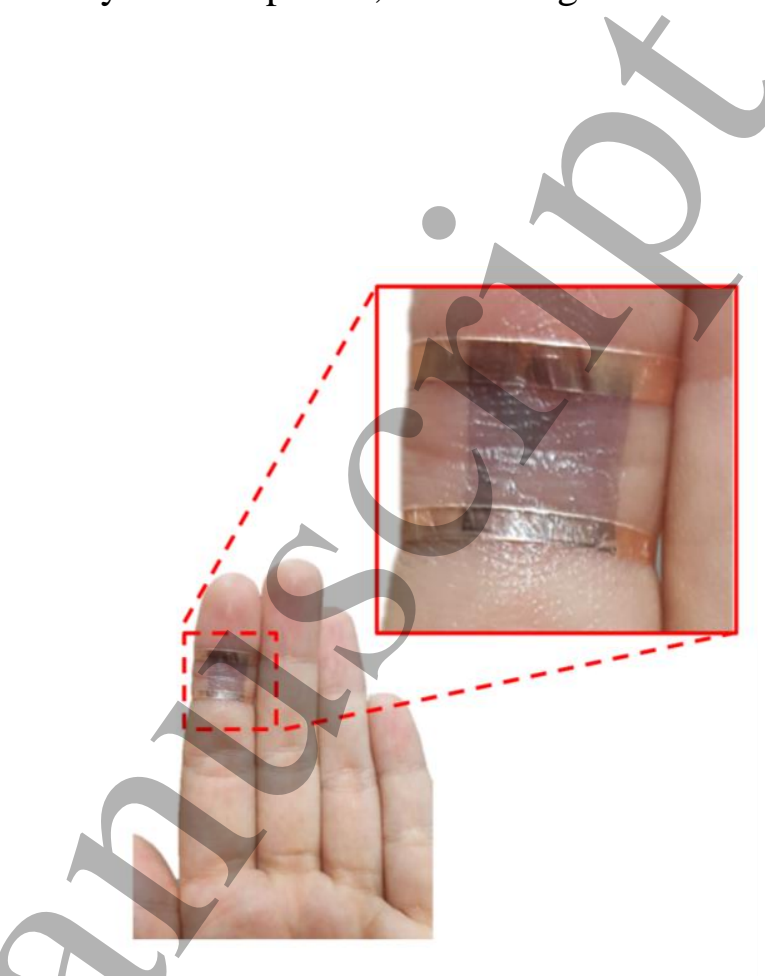

(b)

Figure 3. Evaluation of the resistance of the tattoo electronics on the skin towards bending (a) The change in the electrical resistance of the printed tattoo electrode upon repeated contractions of the index finger at $30^{\circ}$ and $90^{\circ}$ bending angle, (b) Photograph of the tattoo on the index finger of the volunteer and the bending experiment. The tattoo electrode is connected to the acquisition system via copper adhesives which were attached underneath the edges of the conducting pattern.

To test the performance of our tattoo/textile electronics in acquiring EMG signals, we transferred the tattoo onto the forearm of a volunteer and connected the tattoo electrode to the acquisition system using the electronic wristband as shown in Figure 4a. The tattoo electrodes (working and counter) were positioned $2 \mathrm{~cm}$ apart from each other on the lower part of the left arm's biceps and EMG signals were recorded over 6 hours once in every 3 hours. The signals acquired with the tattoo/textile electronics were compared to those acquired with wet $\mathrm{Ag} / \mathrm{AgCl}$ electrodes. These electrodes were positioned at the exact same locations, this time on the right arm which was exposed to the same experimental conditions. 


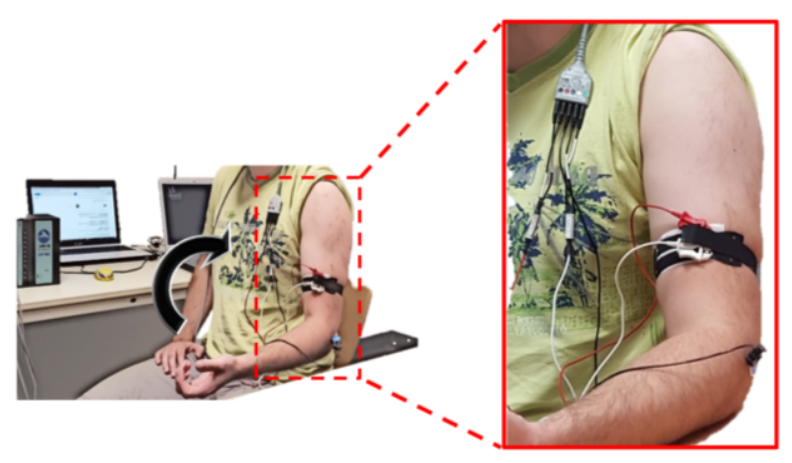

(a)

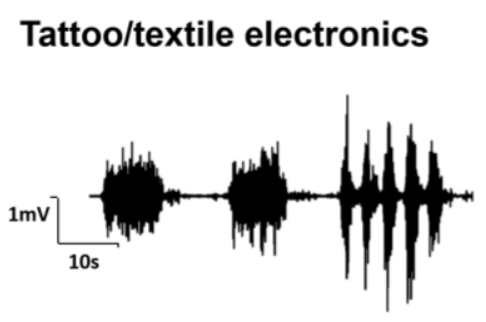

To

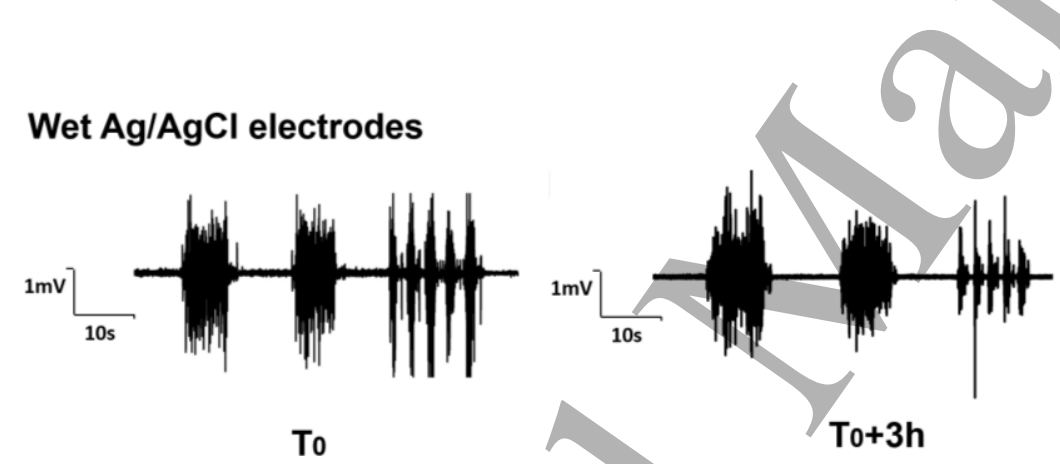

(b)

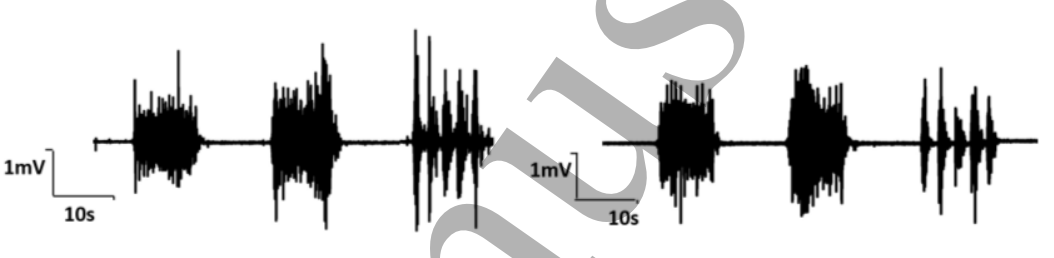

To+6h

Figure 4. EMG recordings of the biceps contraction (a) Photograph of the EMG experiment with a loop on the arm of the volunteer: the tattoo electrodes were deposited on the arm and covered by the textile electrode which was placed underneath a black adjustable sweatband, (b) EMG signals recorded with tattoo/textile electronics after 2 long contractions (10 s) and 5 short contractions ( $1 \mathrm{~s})$ at $\mathrm{T}_{0}+1 \mathrm{~h}, \mathrm{~T}_{0}+3 \mathrm{~h}$ and $\mathrm{T}_{0}+6 \mathrm{~h}$ as well as with wet $\mathrm{Ag} / \mathrm{AgCl}$ electrodes. Each experiment was repeated 6 times.

Figure $4 \mathrm{~b}$ shows the EMG signals recorded with tattoo/textile electronics as well as $\mathrm{Ag} / \mathrm{AgCl}$ electrodes during the biceps activity for both short (1 s) and long (10 s) voluntary muscle contractions over 7 hours. The recordings used to extract the signal to noise ratio (SNR) consisted of 10 seconds-long contractions during which the volunteer was asked to lift a $1 \mathrm{~kg}$ of standard weight with an elbow angle of ca. $90^{\circ}$, with a resting period of 10 seconds. As the electrodes were placed on the skin $\left(T_{0}=1 \mathrm{~h}\right)$, the tattoo/textile electronics exhibited a mean SNR of $11 \pm 2 \mathrm{~dB}$ compared to a mean SNR of $29 \pm 1 \mathrm{~dB}$ for the wet $\mathrm{Ag} / \mathrm{AgCl}$ 
electrodes. The lower quality of the recordings with the dry tattoo after its adherence with the skin is attributed to the high electrode-skin contact impedance of these electrodes (Figure 2a). Despite the lower SNR, the tattoo/textiles electronics could measure the EMG activity. Interestingly, after 4 hours of contact with the skin $\left(\mathrm{t}=\mathrm{T}_{0}+3 \mathrm{~h}\right)$, the performance of the electronics was substantially improved: SNR increased to $26 \pm 2 \mathrm{~dB}$ while the $\mathrm{SNR}$ of $\mathrm{Ag} / \mathrm{AgCl}$ electrodes decreased to $25 \pm 1 \mathrm{~dB}$. Note that the signal-to-noise ratio is expected to be slightly higher at the non-dominant side (i.e., left arm for the right-handed volunteers), validated by the tattoo/textile electronics upon $\mathrm{t}=\mathrm{T}_{0}+3 \mathrm{~h}$. We postulate that the quality of the contact of the tattoo/textile electronics with the skin increased due to the sweat-gland activity. The sweat produced by the body wets the tattoo, thus enhances the ionic conductivity at the skin-electrode interface and leads to a decrease of the contact impedance between the skin and PEDOT:PSS [42]. Another factor that contributed to the reduced impedance is the improved quality of contact between the tattoo and the textile. As shown in Figure 1a, there is a thin layer of the ionic conductor, ethyl cellulose, between the two PEDOT:PSS layers (the tattoo and the textile). As the skin gets wet, this layer becomes more permeable to ions allowing the current to pass easier form the skin to the textile contact. Finally, 7 hours after the first recordings $\left(\mathrm{t}=\mathrm{T}_{0}+6 \mathrm{~h}\right)$, the quality of the recordings are similar, i.e., SNR corresponds to $21 \pm 1 \mathrm{~dB}$ and $20 \pm 5 \mathrm{~dB}$, for tattoo/textile electronics and $\mathrm{Ag} / \mathrm{AgCl}$ electrodes, respectively. To demonstrate the reproducibility of the platform, we recorded similar type of EMG signals from 2 other volunteers using new pairs of printed electrodes as well as at different contraction levels. At $t=\mathrm{T}_{0}+6 \mathrm{~h}$, the SNR values of tattoo/textile electronics and wet $\mathrm{Ag} / \mathrm{AgCl}$ electrodes were $31 \pm 6 \mathrm{~dB}$ vs $30 \pm 4 \mathrm{~dB}$ for one volunteer and $23 \pm 1 \mathrm{~dB}$ vs $21 \pm 1 \mathrm{~dB}$ for the other, respectively (Figure S4). We thus conclude that for recording cutaneous EMG signals, the tattoo/textile electronics show similar performance to clinical skin-contact electrodes.

In order to decouple the effect of the tattoo electrodes from the textile, the EMG signals were recorded using the textile electrodes (Figure S5). Although at $\mathrm{T}_{0}$, the SNR of the textile electrodes was higher than the tattoo/textile electronics, and over time, the textile recordings became less stable (e.g. $18 \pm 2 \mathrm{~dB}$ at $\mathrm{T}_{0}$ 
and $4 \pm 3 \mathrm{~dB}$ at $\left.\mathrm{T}_{0}+6 \mathrm{~h}\right)$. Note that to be able to acquire these signals, the textile band has to be strongly tied around the arm to assure contact with the skin. We, therefore attribute the decrease in the SNR (or its instability) to the low skin-electrode contact quality rather than a degradation of the electrode. These results demonstrate the ability of the tattoo/textile electronics to record stable EMG/signals over time alongside the simplicity of device fabrication/integration and gel electrolyte-free use.

\section{Conclusions}

We designed an inkjet-printed tattoo/textile electronics system utilizing the biocompatible conducting polymer PEDOT:PSS both as the transducer and the contact to the acquisition system. The polymer printed on the tattoo showed excellent adherence to the skin with no evident side effects such as skin irritation or allergies. The electrodes exhibited sufficient conductivity for acquiring muscle activities from the skin. The same conducting polymer was printed on a textile used as a wristband around the skin and acted as a bridge between the tattoo electrode and the external electronics. We show that over 7 hours of EMG recordings (biceps contraction), the tattoo/textile electronics showed similar performance to conventional wet $\mathrm{Ag} / \mathrm{AgCl}$ electrodes without the use of gel electrolytes. The integration of the conducting polymer both as the electrode and contact material minimizes the cost while improving the feasibility of such skinadhering electronics envisaged to be applied for diagnostics and/or therapy. The intimate contact of the tattooed electronics with the skin, its conformability and bending with the movements of the limbs is a great advantage over other cutaneous systems. Our approach for interfacing the external electronics avoids the use of inorganic rigid wires and presents a wearable solution. Due to the versatility of the materials and the processes employed, these systems can encompass a variety of applications such as myoelectric prosthesis or detection of muscle functioning. 
Acknowledgments: The authors thank Dr. Anna Maria Pappa (University of Cambridge) for fruitful discussions and Sky MacPhee and Dr. Alexandra Paterson (KAUST) for proof reading. This work was supported though grants by the ANR and MicroVitae Technologies.

\section{References}

[1] Gambhir S S, Ge T J, Vermesh O and Spitler R 2018 Toward achieving precision health Sci. Transl. Med. 10 eaao3612

[2] Yeo W-H, Kim Y-S, Lee J, Ameen A, Shi L, Li M, Wang S, Ma R, Jin S H, Kang Z, Huang Y and Rogers J A 2013 Multifunctional Epidermal Electronics Printed Directly Onto the Skin Adv. Mater. $252773-8$

[3] J. Bandodkar A, S. Hung V W, Jia W, Valdés-Ramírez G, R. Windmiller J, G. Martinez A, Ramírez J, Chan G, Kerman K and Wang J 2013 Tattoo-based potentiometric ion-selective sensors for epidermal pH monitoring Analyst 138 123-8

[4] Kim J, Valdés-Ramírez G, Bandodkar A J, Jia W, Martinez A G, Ramírez J, Mercier P and Wang J 2014 Non-invasive mouthguard biosensor for continuous salivary monitoring of metabolites Analyst 139 1632-6

[5] Parrilla M, Cánovas R, Jeerapan I, Andrade F J and Wang J 2016 A Textile-Based Stretchable Multi-Ion Potentiometric Sensor Adv. Healthc. Mater. 5 996-1001

[6] Imani S, Bandodkar A J, Mohan A V, Kumar R, Yu S, Wang J and Mercier P P 2016 A wearable chemical-electrophysiological hybrid biosensing system for real-time health and fitness monitoring Nat. Commun. 711650

[7] Tee B C-K, Wang C, Allen R and Bao Z 2012 An electrically and mechanically self-healing composite with pressure- and flexion-sensitive properties for electronic skin applications Nat. Nanotechnol. 7 825-32

[8] Wang S, Xu J, Wang W, Wang G-J N, Rastak R, Molina-Lopez F, Chung J W, Niu S, Feig V R and Lopez J 2018 Skin electronics from scalable fabrication of an intrinsically stretchable transistor array Nature

[9] N, Mazzaro 2016Risk of skin/reaction when using ECG electrodes

[10] Tao X M 2001 Smart Fibres, Fabrics and Clothing: Fundamentals and Applications (Elsevier)

[11] Park S and Jayaraman S 2003 Smart textiles: Wearable electronic systems MRS Bull. 28 585-91

[12] Matsuhisa N, Kaltenbrunner M, Yokota T, Jinno H, Kuribara K, Sekitani T and Someya T 2015 Printable elastic conductors with a high conductivity for electronic textile applications Nat Commun 6 
[13] Yapici M K, Alkhidir T, Samad Y A and Liao K 2015 Graphene-clad textile electrodes for electrocardiogram monitoring Sens. Actuators B Chem. 221 1469-74

[14] Wagner S, Lacour S P, Jones J, Pai-hui I H, Sturm J C, Li T and Suo Z 2004 Electronic skin: architecture and components Phys. E Low-Dimens. Syst. Nanostructures 25 326-34

[15] Kim D-H, Lu N, Ma R, Kim Y-S, Kim R-H, Wang S, Wu J, Won S M, Tao H, Islam A, Yu K J, Kim T -i., Chowdhury R, Ying M, Xu L, Li M, Chung H-J, Keum H, McCormick M, Liu P, Zhang Y-W, Omenetto F G, Huang Y, Coleman T and Rogers J A 2011 Epidermal Electronics Science 333 838-43

[16] Huang X, Liu Y, Cheng H, Shin W-J, Fan J A, Liu Z, Lu C-J, Kong G-W, Chen K, Patnaik D, Lee S-H, Hage-Ali S, Huang Y and Rogers J A 2014 Materials and Designs for Wireless Epidermal Sensors of Hydration and Strain Adv. Funct. Mater. 24 3846-54

[17] Kim J, Banks A, Cheng H, Xie Z, Xu S, Jang K-I, Lee J W, Liu Z, Gutruf P, Huang X, Wei P, Liu F, Li K, Dalal M, Ghaffari R, Feng X, Huang Y, Gupta S, Paik U and Rogers J A 2015 Epidermal Electronics with Advanced Capabilities in Near-Field Communication Small 11 906-12

[18] Miyamoto A, Lee S, Cooray N F, Lee S, Mori M, Matsuhisa N, Jin H, Yoda L, Yokota T, Itoh A, Sekino M, Kawasaki H, Ebihara T, Amagai M and Someya T 2017 Inflammation-free, gaspermeable, lightweight, stretchable on-skin electronics with nanomeshes Nat. Nanotechnol. 12 907-13

[19] Inal S, Rivnay J, Suiu A-O, Malliaras G G and McCulloch I 2018 Conjugated polymers in bioelectronics Acc. Chem. Res. 51 1368-76

[20] Elschner A, Kirchmeyer S, Lovenich W, Merker U and Reuter K 2010 PEDOT: principles and applications of an intrinsically conductive polymer (CRC Press)

[21] Pingree S C L, MacLeod A B and Ginger D 2008 The Changing Face of PEDOT:PSS Films: Substrate, Bias, and Processing Effects on Vertical Charge Transport J. Phys. Chem. C $1127922-$ 27

[22] Mannerbro R, Ranlöf M, Robinson N and Forchheimer R 2008 Inkjet printed electrochemical organic electronics Synth. Met. $158556-60$

[23] Vuorinen T, Niittynen J, Kankkunen T, Kraft T M and Mäntysalo M 2016 Inkjet-Printed Graphene/PEDOT:PSS Temperature Sensors on a Skin-Conformable Polyurethane Substrate Sci. Rep. 635289

[24] Bihar E, Roberts T, Ismailova E, Saadaoui M, Isik M, Sanchez-Sanchez A, Mecerreyes D, Hervé T, De Graaf J B and Malliaras G G 2017 Fully Printed Electrodes on Stretchable Textiles for LongTerm Electrophysiology Adv. Mater. Technol. 21600251

[25] ElMahmoudy M, Inal S, Charrier A, Uguz I, Malliaras G G and Sanaur S 2017 Tailoring the electrochemical and mechanical properties of PEDOT: PSS films for bioelectronics Macromol. Mater. Eng. 302

[26] Bihar E, Roberts T, Saadaoui M, Hervé T, De Graaf J B and Malliaras G G 2017 Inkjet-Printed PEDOT:PSS Electrodes on Paper for Electrocardiography Adv. Healthc. Mater. 1601167 
[27] Håkansson A, Han S, Wang S, Lu J, Braun S, Fahlman M, Berggren M, Crispin X and Fabiano S 2017 Effect of (3-glycidyloxypropyl) trimethoxysilane (GOPS) on the electrical properties of PEDOT: PSS films J. Polym. Sci. Part B Polym. Phys. 55 814-20

[28] Zucca A, Cipriani C, Tarantino S, Ricci D, Mattoli V and Greco F 2015 Tattoo Conductive Polymer Nanosheets for Skin-Contact Applications Adv. Healthc. Mater. 4 983-90

[29] Zucca A, Yamagishi K, Fujie T, Takeoka S, Mattoli V and Greco F 2015 Roll to roll processing of ultraconformable conducting polymer nanosheets J. Mater. Chem. C 3 6539-48

[30] Bareket L, Inzelberg L, Rand D, David-Pur M, Rabinovich D, Brandes B and Hanein Y 2016 Temporary-tattoo for long-term high fidelity biopotential recordings Sci. Rep. 625727

[31] Pitsalidis C, Pappa A-M, Hunter S, Payne M M, Anthony J E, Anthopoulos T D and Logothetidis S 2015 Electrospray-Processed Soluble Acenes toward the Realization of High-Performance FieldEffect Transistors ACS Appl. Mater. Interfaces 7 6496-504

[32] Scheiblin G, Aliane A, Strakosas X, Curto V F, Coppard R, Marchand G, Owens R M, Mailley P and Malliaras G G Screen-printed organic electrochemical transistors for metabolite sensing $M R S$ Commun. 1-5

[33] Ersman P A, Westerberg D, Tu D, Nilsson M, Ảhlin J, Eveborn A, Lagerlöf A, Nilsson D, Sandberg M and Norberg P 2017 Screen printed digital circuits based on vertical organic electrochemical transistors Flex. Print. Electron. 2045008

[34] Roberts T, De Graaf J B, Nicol C, Hervé T, Fiocchi M and Sanaur S 2016 Flexible Inkjet-Printed Multielectrode Arrays for Neuromuscular Cartography Adv. Healthc. Mater. 5 1462-70

[35] Bihar E, Deng Y, Miyake T, Saadaoui M, Malliaras G G and Rolandi M 2016 A Disposable paper breathalyzer with an alcohol sensing organic electrochemical transistor Sci. Rep. 627582

[36] Batchelor J C and Casson A J 2015 Inkjet printed ECG electrodes for long term biosignal monitoring in personalized and ubiquitous healthcare 2015 37th Annual International Conference of the IEEE Engineering in Medicine and Biology Society (EMBC) 4013-6

[37] Ferrari L M, Sudha S, Tarantino S, Esposti R, Bolzoni F, Cavallari P, Cipriani C, Mattoli V and Greco F 2018 Ultraconformable Temporary Tattoo Electrodes for Electrophysiology Adv. Sci. 5 1700771

[38] Kalra A, Lowe A and Jumaily A A 2016 An Overview of Factors Affecting the Skins Youngs Modulus J. Aging Sci. 4 1-5

[39] Searle A and Kirkup L 2000 A direct comparison of wet, dry and insulating bioelectric recording electrodes Physiol. Meas. 21271

[40] Van Boxtel A 2001 Optimal signal bandwidth for the recording of surface EMG activity of facial, jaw, oral, and neck muscles Psychophysiology 38 22-34

[41] Leleux P, J-M Badier, Rivnay J, Christian B, Thierry H, Chauvel P and Malliaras G G. 2013 Conducting Polymer Electrodes for Electroencephalography Adv. Healthc. Mater. 3 490-3 
[42] Gruetzmann A, Hansen S and Müller J 2007 Novel dry electrodes for ECG monitoring Physiol. Meas. 281375

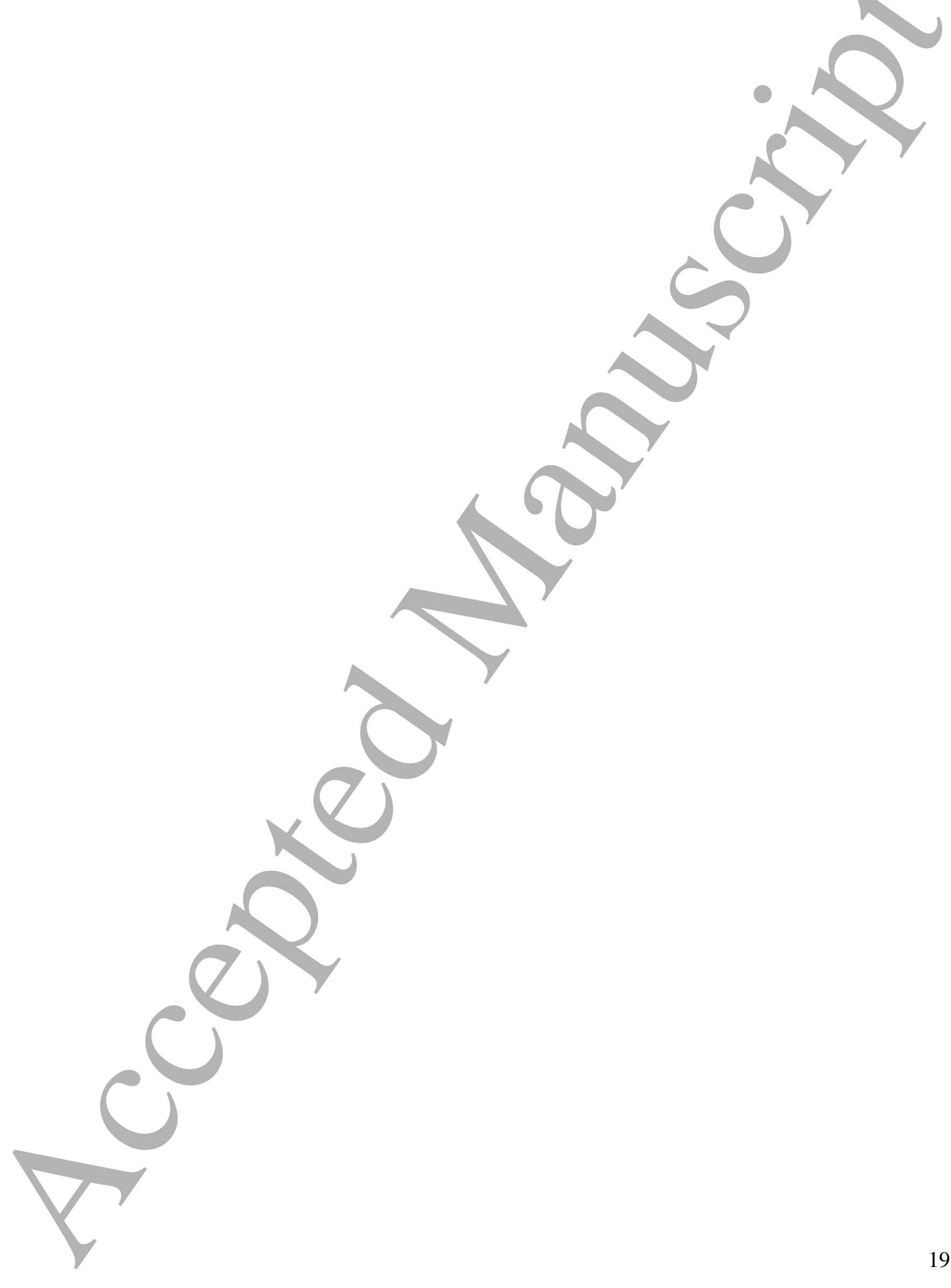

